\title{
Reflets
}

Revue ontaroise d'intervention sociale et communautaire

\section{Ne pas savoir : parlons du mauvais traitement contre les femmes ayant un handicap}

\section{Lynn Lambert}

Volume 4, numéro 2, automne 1998

Personnes vivant avec une incapacité

URI : https://id.erudit.org/iderudit/026223ar

DOI : https://doi.org/10.7202/026223ar

Aller au sommaire du numéro

Éditeur(s)

Reflets : Revue ontaroise d'intervention sociale et communautaire

ISSN

1203-4576 (imprimé)

1712-8498 (numérique)

Découvrir la revue

Citer cet article

Lambert, L. (1998). Ne pas savoir : parlons du mauvais traitement contre les femmes ayant un handicap. Reflets, 4(2), 132-143.

https://doi.org/10.7202/026223ar

Tous droits réservés (C) Reflets : Revue ontaroise d'intervention sociale et communautaire, 1998
Ce document est protégé par la loi sur le droit d'auteur. L'utilisation des services d'Érudit (y compris la reproduction) est assujettie à sa politique d'utilisation que vous pouvez consulter en ligne.

https://apropos.erudit.org/fr/usagers/politique-dutilisation/ 


\section{Ne pas savoir : parlons du mauvais traitement contre les femmes ayant un handicap}

\section{Lynn Lambert \\ Travailleuse sociale, Sudbury}

Il ne s'agit pas d'un projet comme les autres. Au départ, nous avions l'intention d'éduquer et de conscientiser les professionnels de la santé de la région de Sudbury, aux problèmes vécus par les femmes francophones de la région de Sudbury ayant un handicap. Pour ce faire, nous avons dépouillé la littérature les concernant pour nous apercevoir assez rapidement qu'il n'y avait pas de recherche dans les domaines médical ou social portant sur leurs vécus. Ce manque d'informations est à l'origine de la modification de notre projet initial. Il fallait remédier à cette situation et chercher à cerner leurs expériences. C'est pourquoi nous avons approché les femmes francophones de la région ayant un handicap pour qu'elles nous disent leurs attentes et compiler un recueil de leurs expériences. Ce n'est qu'une fois cette tâche accomplie que nous pourrions rencontrer les professionnels de la santé pour leur dire les besoins de cette population. Ainsi, afin de rencontrer notre nouvel objectif, nous avons divisé le projet en trois sections :

1) rechercher les besoins et les expériences,

2) informer les professionnels de la santé et,

3) développer des outils d'information.

Ce projet a eu lieu sous l'égide du Centre de santé communautaire de Sudbury (CSCS) dont la mission est d'aider la population francophone de la région de Sudbury à atteindre un niveau optimal 
de bien-être. Ce bien-être comprend l'aspect physique, mental, économique et social, tant sur le plan individuel que collectif, dans un contexte communautaire. Afin d'atteindre cet objectif, le CSCS favorise une approche multidisciplinaire. C'est précisément dans ce contexte que nous avons élaboré le projet «Ne pas savoir : parlons du mauvais traitement contre les femmes ayant un handicap».

\section{La recherche des besoins et des expériences}

Nous avons effectué 28 entrevues auprès de femmes ayant un handicap à l'aide d'un questionnaire. La première section du questionnaire portait sur des renseignements sociodémographiques (âge, type d'habitation, état civil, éducation, mode de transport, genre d'incapacité, appareils d'aide technique, langue parlée de la répondante, langue maternelle du père ou de la mère). Les femmes avaient des handicaps multiples (spina bifida, paralysie cérébrale, dystrophie musculaire, sclérose en plaque, trouble de vision, surdité, épilepsie, diabète, arthrite (ostéoporose), état maniaco-dépressif, hémophilie, fatigue chronique, polio (syndrome de post-polio). Elles étaient âgées entre 16 et 84 ans et vivaient toutes dans la grande région de Sudbury.

Dans la première section du questionnaire, nous n'avons pas inclus de questions portant sur le revenu des femmes. Cet aspect ne nous semblait pas important lorsque nous avons débuté notre recherche.Toutefois, dans une prochaine recherche, les chercheurs devraient se pencher sur cet aspect afin de mieux le documenter, car certaines indications émises par nos répondantes tendent à dire que les revenus des femmes ayant un handicap sont souvent inférieurs à celui des autres femmes.

Il faudrait aussi s'intéresser aux problèmes vécus par les femmes ayant un handicap dans leur recherche d'emploi. Il s'agirait de voir quels types d'emplois leur sont accessibles et connaitre le genre d'employeurs prêts à les accommoder ${ }^{1}$. Une des pistes de 
solutions à ces difficultés serait de promouvoir l'estime de soi chez les personnes handicapées de telle sorte que certaines d'entre elles puissent devenir entrepreneures. De plus, ces données permettraient de sensibiliser la population aux difficultés rencontrées par les personnes vivant une incapacité dans ce domaine.

La deuxième section du questionnaire portait sur les expériences personnelles des femmes auprès des professionnels de la santé. Ce sont ces expériences tantôt positives, tantôt négatives que nous relaterons dans la suite de ce texte. Pour ce faire, nous avons regroupé les propos des femmes selon le type de professionnels de la santé rencontrés.

\section{a) Médecins, infirmiers et infirmières}

En général, les femmes se sentaient à l'aise avec les médecins. Elles jugeaient les soins adéquats, même si certains médecins cherchaient trop rapidement à prescrire des médicaments plutôt qu'essayer de comprendre la source de leurs maux et de leurs troubles.

Des fois, je rentre voir mon docteur, pis avant que je lui dise où j'ai mal, il me donne des médicaments pour le mal.J'aimerais bien qu'il regarde pourquoi j'ai mal et non assumer que c'est relié à mon handicap (45 ans, fatigue chronique et état manico-dépressif).

La plus grande source de mécontentement exprimée par les femmes provenaient de leurs expériences avec le personnel infirmier et les hôpitaux. Nous en avons conclu que les femmes avaient des contacts plus fréquents avec le personnel infirmier qu'avec les médecins, ce qui expliquerait les mauvaises expériences dont les femmes nous ont fait part.

C'est toujours l'infirmière que je vois. Je demande à voir le médecin, pis l'infirmière dit toujours qu'elle lui a parlé pour moi et qu'il prescrit ceci et cela. J'aimerais bien lui en parler moi-même. Comment est-ce qu'elle sait ce que je veux lui dire? (32 ans, spina-bifida). 
Certaines femmes interviewées établissent un lien entre le manque de service ou la mauvaise qualité des soins qu'elles reçoivent des infirmières et les coupures budgétaires provinciales dans le domaine de la santé.

C'est la faute du gouvernement, oui! Mais les infirmières ont encore le devoir de me soigner et de ne pas faire de fautes quand elles me donnent des médicaments et de ne pas me faire attendre une heure pour aller à la toilette. Par ce temps là, j'avais déjà pissé au lit, pis elle était fâchée contre moi. Pour le restant de mon hospitalisation, $j$ 'avais des couches et j'étais humiliée. Mes enfants et mon mari ne savaient pas comment me soulager. C'est seulement après $d u$ counselling que je me suis sentie mieux et que je me suis plainte à la direction de l'hôpital du mauvais traitement que les infirmières m'avaient fait. Je suis tannée d'entendre que c'est à cause des coupures budgétaires, ce n'est pas mon problème. Et je n'ai pas demandé de me faire traiter comme une enfant (54 ans, sclérose en plaques).

La majorité des femmes (21 femmes au total) se plaignait du manque de service reçu par le personnel infirmier en disant qu'il offrait le minimum des soins, qu'il ne prenait pas le temps de les aider avec leurs besoins complexes (aller à la toilette, se vêtir ou se déshabiller, entrer et sortir du lit, se nourrir, se laver, boire de l'eau, etc.). Si certaines ont exprimé leur gêne d'être soignées par du personnel infirmier masculin, pour d'autres, ce fut des soins donnés avec grande dignité.

Je suis surprise de dire que mes meilleurs soins venaient des infirmières en stage et des infirmiers. Les jeunes infirmières en stage voulaient faire sûre que mes besoins étaient remplis. Elles prenaient le temps quand je devais aller à la toilette ou quand je voulais sortir et entrer $d u$ lit. Mais ce qui m'a le plus surpris, ce sont les soins que j'ai eu des infirmiers. Quand, après ma chirurgie, 
je devais aller à la toilette, les infirmiers étaient les seuls qui prenaient le temps de mettre une serviette sur moi pour me donner un peu de dignité. De plus, quand ils devaient changer mes pansements, ils faisaient sûre que les pansements (ou la colle du pansement) ne touchaient pas les points de sutures. Mais qu'importe! Tu auras toujours des bonnes et des mauvaises infirmières ou même des infirmiers (27 ans, dystrophie musculaire).

\section{b) Préposés aux soins personnels}

La tâche de préposés aux soins personnels n'est pas nouvelle dans le domaine de la santé. Pourtant, en raison de toutes les restructurations et les changements qui se produisent dans les hôpitaux, leur rôle a pris de plus en plus d'importance afin de garantir la qualité des soins que reçoit une femme ayant un handicap. Dans les hôpitaux, institutions ou dans les services de soins communautaires, les préposés font un travail précieux que les infirmières n'ont pas le temps ou ne prennent pas le temps d'accomplir.

Les préposés ne sont pas gênés de m'amener à la toilette et à m'aider à m'habiller. Comme ça mon mari ou mes enfants n'ont pas besoin de le faire! C'est comme si j'étais encore capable de le faire toute seule (45 ans, sclérose en plaques).

De plus, plusieurs femmes prétendent que les préposés prenaient le temps de discuter avec elle ou continuaient à travailler au-delà de leurs temps de visite réglementaire.

Je suis encore humaine et ma préposée me traite comme une amie (45 ans, sclérose en plaques).

Par contre, certaines manifestent de la crainte à leur égard:

Des fois, j'ai peur de les laisser entrer chez moi. J'ai peur qu'elles vont me voler mes affaires (86 ans, ostéoporose). 


\section{c) Travailleurs sociaux, psychologues et psychiatres}

Les femmes semblaient confuses quant au rôle imparti à un travailleur social, un psychologue ou un psychiatre. D'après elles, ces trois types professionnels accomplissent le même travail, celui de fournir de l'aide afin de surmonter et d'accepter les situations difficiles de la vie. En général, l'information recueillie était très positive. L'aide reçue par l'un ou l'autre de ces professionnels avait été grandement appréciée à des moments critiques de la vie (moment de dépression, isolement des mères seules, divorce, mort d'un partenaire, problème avec le mariage ou avec les enfants, changement de l'état de santé, etc.). Le seul point négatif concernant ces professionnels était le manque de personnel féminin et francophone, surtout dans le domaine de la psychologie ou de la psychiatrie.

Je ne parle pas l'anglais. Pis je venais d'avoir mon diagnostic de sclérose en plaques. On a déménagé à Sudbury après avoir vécu au Québec toute notre vie. Je voulais parler avec quelqu'un de mes problèmes de santé, de ma dépression. Mon mari n'était pas content avec son nouveau travail, pis mes enfants voulaient retourner au Québec. J'ai été quelque part, pis la conseillère parlait le français. Mais, elle parlait toujours en anglais. Elle me demandait souvent ce que je voulais dire par mes expressions. Finalement, elle m'a demandé si je pouvais m'exprimer en anglais parce qu'elle ne me comprenait pas en français. Après trois sessions, elle m'a dit que mon problème est que je ne comprends pas et ne parle pas l'anglais. Elle m'a donné une adresse où je pourrais apprendre l'anglais. J'avais pas besoin de ca! Après deux ans, j'ai trouvé une travailleuse sociale qui parlait vraiment le français et qui le comprenait aussi. Mon problème n'était pas la langue mais mon trouble avec l'adaptation du déménagement et $d u$ choc entre la culture québécoise et ontarienne. Et aussi mon nouveau handicap (56 ans, sclérose en plaques, diabète). 
Par ailleurs, et un peu pour répondre à leur confusion initiale, les femmes ont exprimé le besoin d'avoir une définition écrite pour clarifier les rôles des travailleurs sociaux, des psychologues et des psychiatres ainsi qu'une clarification des approches utilisées par chaque profession.

\section{d) Hôpitaux}

Avec les changements structuraux dans les hôpitaux de Sudbury, les femmes francophones ayant un handicap sont très craintives à l'idée d'être hospitalisées et de ne pas recevoir les soins adéquats. La plupart des expériences touchaient le personnel infirmier, comme nous l'avons dit plus haut. Pourtant, plusieurs jugent que les coupures budgétaires ont eu un impact négatif sur les soins dans les hôpitaux. Plusieurs femmes ont mentionné que le personnel infirmier n'avait plus le temps ou ne prenait pas le temps de les écouter et comprendre leurs besoins. De plus, les hôpitaux devraient être plus sensibles aux traitements que reçoivent les femmes ayant un handicap lorsqu'elles sont hospitalisées. Par exemple, il ne faudrait pas mettre dans une même chambre une femme avec un handicap physique de 27 ans avec une femme de 75 ans qui souffre de la maladie d'Alzheimer.

J'avais 27 ans et j'étais à l'hôpital parce que j'avais des plaies sur le derrière et dans le dos. On m'a mise dans une chambre double avec une vieille femme de 75 ans. La femme avait l'Alzheimer. Elle criait souvent et ne cessait pas de pleurer. J'avais demandé de changer de chambre et l'infirmière du plancher m'a dit qu'il n'y avait pas d'autres lits ou chambres libres. Durant le milieu de la nuit, je me suis réveillée parce que quelqu'un était dans mon lit et essayait de me pousser de mon lit. J'ai appelé pour de l'assistance, mais cela a pris environ trente minutes pour qu'une infirmière vienne. La femme de 75 ans était dans mon lit et voulait mon lit. Les infirmières étaient incapables de la faire changer d'idée. Finalement, après 
deux heures dans un lit avec une femme qui criait et pleurait, on m'a finalement fait sortir du lit (j'étais incapable de le faire moi-même) et on m'a mis dans une autre chambre. Cette expérience m'a traumatisée. Après deux jours et plusieurs plaintes à l'administration de l'hôpital, je suis sortie de l'hôpital et j'ai demandé de recevoir des soins à la maison. Cela m'a pris environ trois ou quatre mois pour m'en remettre. Les cris, je les entends encore parfois lorsque je dors (32 ans, spina-bifida).

Selon cette femme, l'hôpital a une responsabilité de protéger ses patients ou ses patientes. À cette fin, il ne faudrait pas prendre pour acquis que tous les handicaps sont pareils, car chaque handicap est différent (alzheimer ou spina-bifida).

Durant les entrevues, il est apparu évident que les femmes ayant des handicaps sont craintives à l'idée d'être hospitalisées. Elles préfereraient de loin demeurer à la maison et y recevoir des soins à domicile offerts par les infirmières de l'Ordre de Victoria qui sont désormais de plus en plus accessibles.

\section{e) Les organismes à but non lucratif et services communautaires}

Dans notre recherche, nous avons demandé aux femmes de décrire leurs expériences auprès des organismes à but non lucratif et des services communautaires qui desservent les femmes ayant un handicap. Dix organismes ont été mentionnés le plus souvent par les répondantes, soit La marche des dix sous (March of Dime), le Projet de participation communautaire du District de Sudbury, le Centre d'accès aux soins communautaire (Homecare), l'Ordre de Victoria (V.O.N.), la Société de sclérose en plaques, l'Institut national canadien pour les aveugles (I.N.C.A.), les Timbres de Pâques (Easter Seals Society), le Centre de santé communautaire, le Club des veufs et des veuves de Sudbury, les Services de popote roulante (Meals on wheels). Dans cette section, nous résumerons les témoignages des répondantes. 
Les principaux commentaires ont trait à l'accessibilité. En effet, les répondantes notent rapidement le manque d'accès à un édifice et au service qui s'y loge. Cette barrière contribue à juger négativement l'un ou l'autre de ces services. Le même type de jugement catégorique se produit lorsque le personnel des services infantilise la personne ayant une incapacité et essaie de lui dire ce qui est bon pour elle contre son propre avis.

Généralement, les répondantes n'ont pas éprouvé beaucoup de difficultés à obtenir l'information désirée auprès des ressources communautaires. Le problème apparaît lorsqu'il est question d'argent. Pour bénéficier d'un service ayant des aspects financiers, les personnes ayant une incapacité doivent satisfaire à un nombre de critères très stricts qui varient d'un service à l'autre. Cela est source d'énormes frustrations. En effet, on publicise qu'un service peut vous venir en aide, pour s'apercevoir une fois sur place qu'on ne répond pas aux critères. Cela est d'autant plus frustrant que ce coup de pouce peut être salutaire pour des personnes qui vivent pauvrement.

Rien ne remplace la qualité et la chaleur de bons rapports humains. Les répondantes sont unanimes à dire que leur appréciation d'un service en dépend largement. L'écoute attentive de leurs besoins, le dévouement du personnel à leur égard contribuent à l'appréciation positive du service. À l'opposé, lorsqu'on vous traite comme un numéro, qu'on ne tient pas compte de vos demandes, cela vous laisse un goût amer et vous juger négativement l'agence.

\section{Sessions d'information pour les professionnels de la santé}

Nous avons tenu deux sessions d'information à l'intention des professionnels de la santé, à la fin du mois de juillet 1998. Le nombre espéré de participants par session était de 12 à 15 personnes. Or, à nos deux sessions, nous avons malheureusement 
réuni très peu de participants, soit trois pour la première et quatre pour la seconde. Les sessions étaient d'une durée d'une heure et demie. Nous avons tenu à ce que les sessions soient les plus informatives et constructives que possible.

\section{Outils d'information}

Notre projet avait pour but d'élaborer des outils d'information pour sensibiliser les professionnels de la santé et le public aux conditions vécues par les personnes ayant une incapacité en matière de santé. Le premier de ces outils a été la production d'une vidéo d'une heure. Elle comporte des extraits de la première session d'information, ainsi que les communications de trois participantes (infirmière médicale, travailleuse sociale et infirmière spécialisé en développement communautaire). On peut emprunter la vidéo auprès de la bibliothèque du Centre de santé communautaire de Sudbury.

Le second outil a trait à la création d'une page WEB. Le but de la page WEB sera de rendre accessible à la communauté l'information recueillie pendant le projet. On pourra y trouver une brève description du Centre de santé communautaire de Sudbury, les objectifs de la recherche et surtout, les ressources communautaires (agences ou groupes) qui offrent des services aux femmes francophones ayant un ou des handicaps. De plus, nous prévoyons ajouter une liste des endroits et des édifices accessibles pour celles qui ont des problèmes de mobilité.

Durant nos sessions d'information, il est devenu de plus en plus évident que les femmes ne connaissaient pas toujours les rôles des différents professionnels de la santé. Nous avons donc décidé de créer, comme troisième outil d'information, un dépliant décrivant le Centre de santé communautaire et la description de tâches des divers professionnels qui y travaillent. 


\section{Les limites de notre recherche}

Le temps a été un facteur important dans le développement de notre projet. Il faut dire que nos répondantes ont exprimé beaucoup de réticences à l'idée de nous confier leurs expériences. Cette réticence est compréhensible, si nous pensons aux nombreuses formes d'intrusions qu'elles vivent constamment lors d'examens physiques, de sessions de thérapies ou encore, lorsqu'elles consultent un conseiller. Elles ne se prêtent donc pas volontiers à une nouvelle invasion de leurs vies. Bien que nous ayons commencé notre projet au début du mois de mai, nous n'avons pu réaliser notre première entrevue qu'à la fin du mois de juin. La résistance des femmes ayant un handicap est grande à l'idée de parler des mauvais traitements qu'elles subissent. Et elle est souvent liée à la peur de perdre les services.

De plus, nos démarches auprès des agences, des organismes et des services qui travaillent régulièrement auprès des personnes ayant un handicap ont demandé beaucoup de temps et d'énergie. Au départ, nous avions demandé à ces services d'informer leurs clientèles francophones et féminines de l'existence de notre projet de recherche et de leur indiquer que nous étions à la recherche de participantes. Cette démarche n'a pas eu la réponse escomptée. Nous avons alors opté pour une approche plus directe en rencontrant la clientèle des ces services. Toutefois, les nombreux contacts qu'une telle démarche a nécessités ont fait perdre un temps précieux, bien qu'il fut récompensé. En effet, cinq personnes supplémentaires se sont jointes à notre projet de recherche.

La période de l'année à laquelle notre recherche et nos sessions d'information ont eu lieu, a été peu propice. En effet, une grande partie des professionnels de la santé était en vacances. Lors des sessions d'information, plusieurs travailleurs sociaux qui nous avaient signifié un intérêt pour y participer, se trouvaient en vacances. Le même phénomène s'est reproduit avec les médecins. Ainsi, malgré nos efforts pour réunir le grand nombre de professionnels de la santé, il est devenu évident que la période 
choisie de nos sessions d'information n'était pas la bonne et que les mois de septembre, octobre et novembre auraient été de loin préférables.

Cela dit, ces facteurs n'expliquent pas tout. Tant du coté des professionnels de la santé que de celui des femmes elles-mêmes, il existe un malaise à aborder ouvertement la question du mauvais traitement envers les femmes ayant un handicap. En effet, peu de professionnels de la santé sont prêts à admettre que cette réalité existe bel et bien. Par ailleurs, bien que les femmes témoignent des mauvais traitements qu'elles subissent, elles invoquent toutes sortes d'excuses pour l'expliquer. Nous nous demandons si un tel phénomène n'est pas attribuable à l'extrême dépendance physique, économique et sociale que vit la majorité des femmes ayant un handicap. Par ailleurs, nous regrettons amèrement que la direction de certains hôpitaux ait refusé de nous laisser faire nos présentations auprès des infirmières et des infirmiers sous prétexte qu'elles se dérouleraient en français.

Le projet est terminé. Pourtant, nous aurions aimé tenir plus de sessions d'information et faire plus de présentations afin de sensibiliser les professionnels de la santé aux besoins des femmes francophones ayant un handicap. Cela dit, en terminant,j'aimerais formuler quelques souhaits propres à accroître cette sensibilité aux besoins de ces femmes. Dans la région de Sudbury, il serait souhaitable d'avoir plus d'informations en français sur les différents handicaps et que celles-ci soient accessibles à un large public et aux professionnels de la santé. De plus, nous aimerions voir la création d'un guichet unique, dévolu à une agence, pour tous les services touchant les personnes ayant un handicap.

\section{Note}

1. Voir l'article de Barette dans ce numéro qui évoque cet aspect (ndlr). 